\title{
Mass shift of sigma-Meson in Nuclear Matter
}

\author{
Article in Pramana - March 2013
}

DOI: $10.1007 /$ s12043-012-0503-2 · Source: arXiv

CITATIONS

0

4 authors, including:

\section{Jose Ruben Morones Ibarra}

Autonomous University of Nuevo León

40 PUBLICATIONS 119 CITATIONS

SEE PROFILE

\section{Felipe Robledo-Padilla}

Tecnológico de Monterrey

5 PUBLICATIONS 3 CITATIONS

SEE PROFILE
READS

54

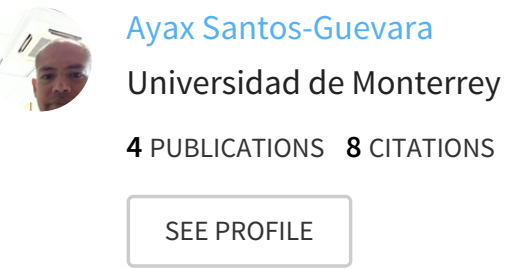

Some of the authors of this publication are also working on these related projects: 


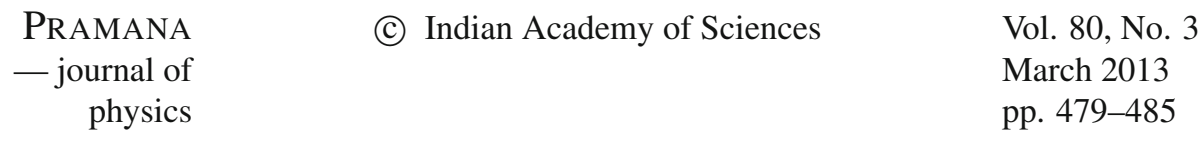

\title{
Mass shift of $\sigma$-meson in nuclear matter
}

\author{
J R MORONES-IBARRA ${ }^{1}$, MÓNICA MENCHACA MACIEL ${ }^{1, *}$, \\ AYAX SANTOS-GUEVARA ${ }^{2}$ and FELIPE ROBLEDO PADILLA ${ }^{1}$ \\ ${ }^{1}$ Facultad de Ciencias Físico-Matemáticas, Universidad Autónoma de Nuevo León, UANL, \\ Av. Universidad S/N Ciudad Universitaria, San Nicolás de los Garza, Nuevo León, \\ C.P. 66451 , México \\ ${ }^{2}$ Facultad de Ingeniería y Arquitectura, Universidad Regiomontana, 15 de Mayo 567, Monterrey, \\ N.L., 64000, México \\ *Corresponding author. E-mail: monica.menchaca@uanl.mx
}

MS received 13 July 2011; revised 8 March 2012; accepted 22 August 2012

\begin{abstract}
The propagation of $\sigma$-meson in nuclear matter is studied in the Walecka model, by assuming that the sigma couples to a pair of nucleon-antinucleon states and to particle-hole states. The in-medium effect of $\sigma-\omega$ mixing is also studied. For completeness, the coupling of sigma to two virtual pions was also considered. It is found that the $\sigma$-meson mass decreases with respect to its value in vacuum and that the contribution of the $\sigma-\omega$ mixing effect on the mass shift is relatively small.
\end{abstract}

Keywords. Scalar mesons; hadrons in dense matter; spectral function; dense nuclear matter.

PACS Nos 13.75.Lb; 14.40; 14.40Cs; 21.65.+f

\section{Introduction}

The study of matter under extreme conditions of density and temperature has become very important because it prepares one to understand the physics for some interesting topics, such as the conditions in the early Universe, the physics of processes in stellar evolution and in heavy-ion collision. Particularly, the study of properties of mesons in hot and dense matter is significant for comprehending the possible signature for detecting the quark-gluon plasma (QGP) state in heavy-ion collision, as well as to obtain information about the signal of the presence of QGP and also to know which symmetries are restored [1].

The study of meson propagation in a medium is essential to interpret the properties of nuclear matter [2]. In this work, we use the Walecka model to investigate the $\sigma$-meson propagation in nuclear matter. The Walecka model is a renormalizable relativistic quantum field theory for nuclear matter where the degrees of freedom are nucleons 


\section{$J$ R Morones-Ibarra et al}

that interact through the exchange of scalar and vector mesons: the sigma and the omega, respectively $[3,4]$. This model has proved to be very successful in explaining several processes in nuclear theory [5].

Much studies were done on hadrons in dense nuclear matter in the last few years. One of the interesting phenomena of nuclear physics that has been recently explained, fitting the data by the shift-down hypothesis of the meson masses in nuclear matter, is the anomalous long lifetime of the C-14 nucleus [6].

One of the phenomenological reasons to include the $\sigma$-meson as a $\bar{q} q$ degree of freedom is the fact that the linear sigma model has been very successful in reproducing the data for many processes in $\pi N$ and $\pi \pi$ interactions. On the other hand, theoretical predictions and models which have been successful require the existence of the $\sigma$-meson [7]. It plays a fundamental role in understanding the symmetry-breaking mechanism in QCD and also in the confinement problem [8].

The existence of $\sigma$-meson with a mass of about $500 \mathrm{MeV}$ has been the subject of a long-standing controversy. However, recently it was announced that the $\sigma$-meson has been detected in $\pi \pi \rightarrow \pi \pi$ processes and also in the decay products of heavy quark systems [9]. Thus, after the experimental announcement of the existence of $\sigma$-meson, the controversy is not about its existence but about its structure [9]. The composition of $\sigma$ meson as a $\bar{q} q$-meson or as a $\pi \pi$ resonant particle is still a subject of debate [10-13]. We assume in this paper, that the $\sigma$-meson is a two-pion resonance, according to the Particle Data Group Collaboration [14].

It is still an open question whether $\sigma$ is a fundamental particle, or a dynamically generated particle. Today, the discussion about $\sigma$ has moved from the speculation about its existence, to its structure or dynamics [7].

The relevance of $\sigma$-meson in hadron and nuclear physics can be seen from the following phenomena [15]: (a) The $\sigma$-meson is responsible for the attractive part, in the intermediate range, of the nuclear force $[4,16]$. Without this force, we cannot even explain the existence of nuclei, the bound state of nucleons. (b) The $\sigma$-meson participates as an intermediate particle in several nuclear processes and in pion-nucleon scattering [16,17]. (c) In meson-meson interaction, the $\sigma$-meson occurs as a resonance, showing up as a pole in the $T$-matrix $[16,18,19]$. In the sigma model, developed by Gell-Mann and Levy $[16,20]$, the $\sigma$-meson plays a role similar to the Higgs particle in the standard electroweak theory $[16,20,21]$, giving mass to the nucleon when the $S U(2)_{\mathrm{L}} \otimes S U(2)_{\mathrm{R}}$ symmetry is spontaneously broken. Additionally it can be proved, from the quark structure, that the $\sigma$-meson is the chiral partner of the pion [22].

According to ref. [9], the identification of $\sigma$-meson was realized in the experiment $D^{+} \rightarrow \pi^{-} \pi^{+} \pi^{+}$. The experimental results of this three-body decay of charm meson can be fitted by assuming an intermediate scalar-isoscalar state which can be identified with the $\sigma$-meson.

In this work we study the scalar $\sigma$-meson in nuclear medium when the meson couples to two nucleons through a Yukawa-type coupling. The interaction of the meson with nucleons includes interactions with the Fermi sea producing nucleon-hole states and interactions with the Dirac sea producing nucleon-antinucleon states [3,4]. The $\sigma-\omega$ mixing is also considered in the calculations.

We have associated the mass of sigma to the peak position of its spectral function. The $\sigma$-meson self-energy is calculated in the one-loop level and the propagator is computed by 
summing over ring diagrams, in the so-called random phase approximation (RPA) [23]. To carry out the summation, we use the Dyson's equation.

\section{Formalism}

Starting from the Walecka model, we study the possible modifications of the $\sigma$-meson mass in the nuclear matter. For this purpose, interactions of $\sigma$-meson with particlehole states, nucleon-antinucleon excitations in the Dirac sea, and the $\sigma-\omega$ mixing effect [3-5] were considered. The $\sigma-\omega$ mixing is a purely density effect which occurs when a particle-hole pair is excited in the medium, and, as presented in figure 1, the scalar meson decays into a longitudinal $\omega$-meson. After this, the inverse process send it back to the original $\sigma$-meson. In ref. [24], the authors studied the mixing effect in dilepton production in relativistic heavy-ion experiments.

The study of meson propagation in nuclear medium requires the inclusion of the $\sigma-\omega$ mixing effect. In order to evaluate the mass of $\sigma$-meson, we need, firstly, to calculate its dressed propagator. The mixing effect results in the coupling between the Dyson's equation for the sigma and omega propagators. The expression for the full $\sigma-\omega$ propagator can be written as

$$
i \Delta(k)=i \Delta_{0}(k)+i \Delta_{0}(k)[-i \Sigma(k)] i \Delta(k),
$$

where $i \Delta_{0}(k)$ is the non-interacting $\sigma$-meson propagator and is written as [3,5]

$$
i \Delta_{0}(k)=\frac{i}{k^{2}-\left(m_{\sigma}^{0}\right)^{2}+i \varepsilon}
$$

and $\Sigma(k)$ is the $\sigma$-self-energy. The self-energy $\Sigma(k)$ contains all the information about the interaction between the $\sigma$-meson with nucleons and with $\omega$-mesons in nuclear matter. In order to determine the self-energy $\Sigma(k)$, we must specify the dynamical content of our model.

We start with the Lagrangian density for the Walecka model, which is given as [4]

$$
\begin{aligned}
L= & \bar{\Psi}\left[\gamma_{\mu}\left(i \partial^{\mu}-g_{v} V^{\mu}\right)-\left(M-g_{s} \Phi\right)\right] \Psi+\frac{1}{2}\left(\partial_{\mu} \Phi \partial^{\mu} \Phi-\left(m_{\sigma}^{0}\right)^{2}\right) \Phi^{2} \\
& -\frac{1}{4} F_{\mu \nu} F^{\mu \nu}+\frac{1}{2} m_{\nu}^{2} V_{\mu} V^{\mu}+\delta L
\end{aligned}
$$

where $\Psi$ is the nucleon field with mass $M, V^{\mu}$ is the neutral vector meson field $(\omega)$ with mass $m_{v}$, the tensor field of the vector meson is $F^{\mu \nu}=\partial^{\mu} V^{\nu}-\delta^{\nu} V^{\mu}, \Phi$ is the scalar $(\sigma)$ meson field with the bare mass $m_{\sigma}^{0} ; g_{\mathrm{s}}$ and $g_{v}$ are the coupling constants, and finally $\delta L$ contains renormalization counter terms.

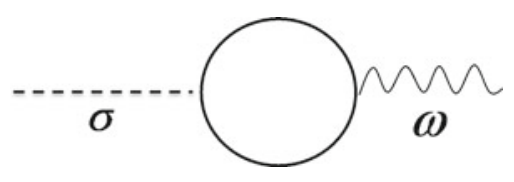

Figure 1. Sigma-omega mixing diagram. 


\section{$J$ R Morones-Ibarra et al}

The interaction Lagrangian density which describes the $\sigma-\pi$ dynamics [25], is given by

$$
L_{\sigma \pi \pi}^{\mathrm{eff}}=\frac{1}{2} g_{\sigma \pi \pi} m_{\pi} \vec{\pi} \cdot \vec{\pi} \Phi
$$

The influence of the interaction of $\sigma$-mesons with nucleons and $\omega$-mesons in nuclear matter is introduced through the modification of the free propagator in the one-loop approximation. We shall calculate the full propagator in the chain approximation, which consists of an infinite summation of the one-loop self-energy diagrams [5]. The diagrammatical representation of the modified propagator is shown in figure 2, and the analytical expression is given by $i \Delta(k)$.

The solution for $\Delta(k)$ in the Dyson's eq. (1) is given by

$$
\Delta(k)=\frac{1}{\Delta_{0}^{-1}(k)-\Sigma(k)}=\frac{1}{k^{2}-\left(m_{\sigma}^{0}\right)^{2}-\Sigma(k)} .
$$

The analytical expression for the self-energy $\Sigma(k)$, is given as [25,26]:

$$
\Sigma(k)=\Sigma_{\sigma \pi}(k)+\Sigma_{\sigma N}(k)+\Sigma_{\mu}^{m}(k),
$$

where the calculation of $\Sigma_{\sigma \pi}(k)$ and $\Sigma_{\sigma N}(k)$ are given in ref. [16] and the self-energy of the $\sigma-\omega$ mixing is given by

$$
i \Sigma_{\mu}^{m}(k)=i g_{s} g_{\nu} \int \frac{\mathrm{d}^{4} q}{(2 \pi)^{4}} \operatorname{Tr}\left[G(q) \gamma_{\mu} G(q+k)\right] .
$$

The scalar-vector mixing effect between $\sigma$ and $\omega$ is a purely medium effect, occuring when a longitudinal $\omega$-meson produces a particle-hole state which decays into a $\sigma$, or vice versa. $G(q)=G_{\mathrm{F}}(q)+G_{\mathrm{D}}(q)$ is the full nucleon propagator [4]:

$$
\begin{aligned}
G(q)= & \left(\gamma_{\mu} q^{* \mu}+M^{*}\right)\left[\frac{1}{q^{* 2}-M^{* 2}+i \varepsilon}\right. \\
& \left.+\frac{i \pi}{E^{*}(q)} \delta\left(q_{0}-E^{*}(q) \Theta\left(k_{\mathrm{F}}-|\vec{q}|\right)\right)\right],
\end{aligned}
$$

where $q^{* \mu} \equiv\left(q^{0}-g_{v} V^{0}, \vec{q}\right), E^{*}(q) \equiv \sqrt{\vec{q}^{2}+M^{* 2}}, k_{\mathrm{F}}$ is the Fermi momentum and $M^{*}$ is the effective mass of the nucleon.

We take as the definition of the mass of a particle, the magnitude of the four-momentum $K$ for which the spectral function gets its maximum, a definition well-established and used

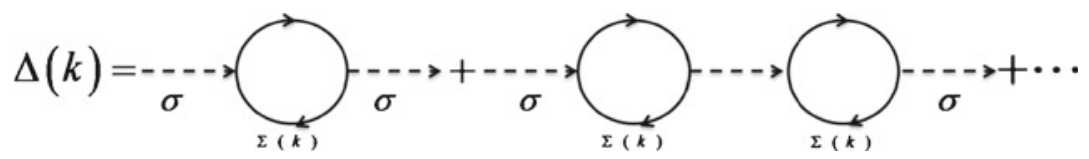

Figure 2. Sigma-meson propagator. 
extensively in literature [27-29]. For this reason, this definition is utilized in this work for calculating the meson mass in nuclear matter.

\section{Calculation of the $\sigma-\omega$ mixing term}

Integrating eq. (8) with respect to $q^{0}$ we obtain

$$
\Sigma_{\mu}^{m}(k)=8 \pi g_{s} g_{\nu} \int \frac{\mathrm{d}^{3} q}{(2 \pi)^{4}} \frac{\left[4 k^{\mu} q^{2}+4 k \cdot q q^{\nu}\right] \Theta\left(k_{\mathrm{F}}-|\vec{q}|\right)}{\left[\left(k^{2}\right)^{2}-4(q \cdot k)^{2}\right] E^{*}(q)} .
$$

On the other hand, as the baryonic current is conserved, it follows that

$$
k^{\mu} \Sigma_{\mu}^{m}(k)=0 .
$$

Taking the condition as for low energies [3] and taking $\vec{k}$ in the positive direction of the $z$-axis, we find

$$
\Sigma_{\mu}^{R m}(k)=\frac{g_{s} g_{v} M^{*}}{\pi^{2}}\left[2 k_{\mathrm{F}}-C_{0} \varepsilon_{\mathrm{F}} \ln \left|\frac{C_{0} \varepsilon_{\mathrm{F}}+k_{\mathrm{F}}}{C_{0} \varepsilon_{\mathrm{F}}-k_{\mathrm{F}}}\right|\right],
$$

where

$$
C_{0}=\frac{k_{0}}{|\vec{k}|} \quad \text { and } \quad \varepsilon_{\mathrm{F}} \equiv \sqrt{k_{\mathrm{F}}^{2}+M^{* 2}}
$$

The propagator takes the form

$$
\Delta\left(k^{2}\right)=\frac{1}{k^{2}-m_{\sigma}^{2}-\operatorname{Re} \Sigma^{R}\left(k^{2}\right)-i \operatorname{Im} \Sigma^{R}\left(k^{2}\right)}
$$

with

$$
\begin{aligned}
& \operatorname{Re} \Sigma^{R}(k)=\operatorname{Re}\left[\Sigma_{\sigma \pi}^{R}(k)+\Sigma_{\sigma N}^{R}(k)+\Sigma_{\mu}^{R m}(k)\right] \\
& \operatorname{Im} \Sigma(k)=\operatorname{Im} \Sigma_{\sigma \pi}(k) .
\end{aligned}
$$

The parameters in eq. (12) are the reported $m_{\sigma}$ value for the $\sigma$-meson mass, which we take in the range $450 \mathrm{MeV}<m_{\sigma}<550 \mathrm{MeV}$, the bare $\sigma \pi \pi$ coupling constant $g_{\sigma \pi \pi}=$ 12.8, the bare $\sigma N$ coupling constant $g_{s}^{2}=54.289$ and the bare $\omega N$ coupling constant $g_{v}^{2}=102.770[5]$.

The spectral function of the $\sigma$-meson at twice the normal nuclear matter density is plotted in figure 3. The nucleon mass was fixed at the physical value $M=939 \mathrm{MeV}$. The effective nucleon mass $M^{*}$ is the appropriate value at nuclear-matter saturation density. This relation was taken as $M^{*} / M=0.730$, and the bare $\sigma N$ coupling constant $g_{s}^{2}=$ 54.289 [5].

As shown in figure 3, the mass of $\sigma$-meson in a medium suffers a down-shift with respect to its mass in vacuum. It is worth saying that there is no unique result concerning the shift mass of mesons in medium. In ref. [2] they obtained an increase in the effective mass of the $\sigma$-meson. On the other hand, authors who have used different models such 


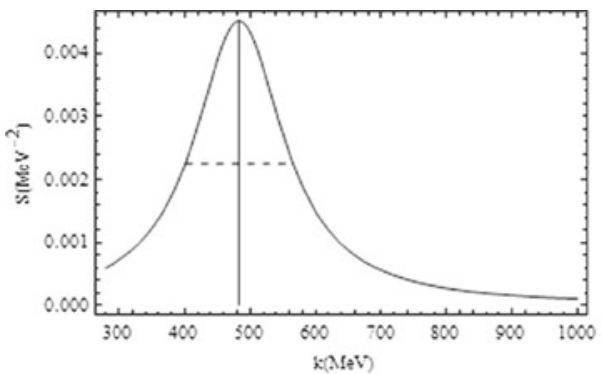

(a)

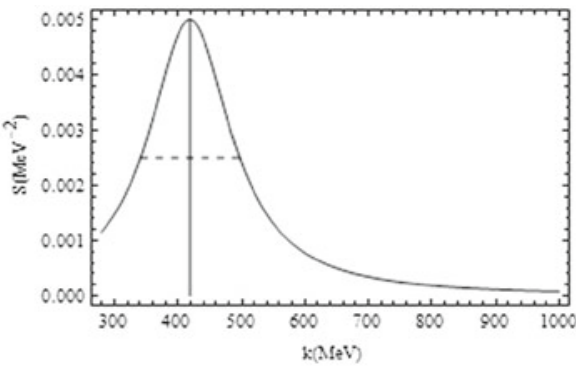

(b)

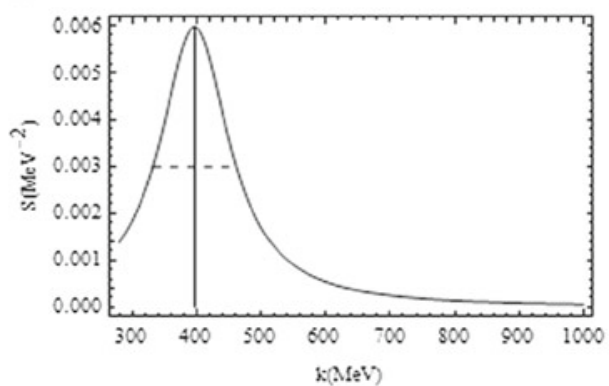

(c)

Figure 3. Spectral function of the $\sigma$-meson at twice the normal nuclear matter: (a) in vacuum, (b) without mixing effect and (c) with mixing effect.

as Nambu-Jona-Lasinio or QCD sum rules, have reported a reduction of the $\sigma$-meson effective mass in medium.

\section{Conclusion}

In the study of the propagation of scalar $\sigma$-meson in nuclear matter, we have found that the effective mass of the meson decreases with respect to its value in vacuum. Assuming that the $\sigma$-meson couples to a particle-hole pair in nuclear matter, that it interacts with nucleon-antinucleon states in the Dirac sea, and also by including, for completeness, the coupling of sigma to two pions, we obtained $13 \%$ reduction in the value of the $\sigma$-meson mass without considering the scalar-vector mixing and $18 \%$ reduction by considering the mixing effect. The mixing effect between $\sigma$-mesons and $\omega$-mesons occurs only in a medium, when a longitudinal $\omega$-meson produces a particle-hole state which decays into a $\sigma$-meson, or vice versa. When this $\sigma-\omega$ mixing is included, we found that the effect is small on the effective mass of the $\sigma$-meson. The effective mass of $\sigma$-meson when the $\sigma-\omega$ mixing effect is included, decreases in a small amount, a difference of $5 \%$ at twice the normal nuclear matter density. These results are in agreement with those obtained by using other models, such as the Nambu-Jona-Lasinio [30,31] and the chiral perturbation theory $[18,19]$, which predict a decrease in the mass of the $\sigma$-meson in a medium. This result can be interpreted as a partial restoration of chiral symmetry $[32,33]$. On the other 


\section{Mass shift of $\sigma$-meson in nuclear matter}

hand, by taking the width at one half of the maximum value of the spectral function, a reduction of $23 \%$ with respect to the width in the vacuum is obtained.

\section{References}

[1] P Costa, M C Ruiro and Yu L Kalinovsky, Phys. Lett. B560, 171 (2003)

[2] J C Caillon and J Labarsouque, Phys. Rev. C59, 1090 (1999)

[3] S A Chin, Ann. Phys. (N.Y.) 108, 301 (1977)

[4] B D Serot and J D Walecka, Adv. Nucl. Phys. 16, 1 (1986)

[5] H C Jean, J Piekarewicz and A G Williams, Phys. Rev. C34, 1981 (1994)

[6] J W Holt, G E Brown, T T Kuo, J D Holt and R Machleidt, Phys. Rev. Lett. 100, 062501 (2008)

[7] E van Beveren, F Kleefeld, G Rupp and M Scandron, Mod. Phys. Lett. A17, 1673 (2002)

[8] N Tornqvist, hep-ph/0204215 (6 June 2002)

[9] E M Aitala et al, Phys. Rev. Lett. 86, 770 (2001)

[10] L Roca and E Oset, Nucl. Phys. A723, 129 (2003)

[11] M Ishida, Prog. Theor. Phys. Suppl. 149, 190 (2003)

[12] N A Tornqvist, in Proc. Possible Existence of Sigma Meson and its Implication to Hadron Physics (Sigma Meson 2000), YITP Kyoto, June 2000, Kyoto 102 No. 5 (2001); KEKProceedings 2000-4

[13] J R Morones-Ibarra and A Santos-Guevara, Acta Phys. Pol. B38, 2555 (2007)

[14] Particle Data Group: C Amsler et al, Phys. Lett. B667, 1 (2008)

[15] T Kunihiro, hep-ph/0705.0072 (1 May 2007)

[16] J R Morones-Ibarra and A Santos-Guevara, Pramana - J. Phys. 68, 925 (2007)

[17] M K Volkov, A E Radzhabov and N L Russakovich, Phys. Atom. Nucl. 66, 997 (2003)

[18] J A Oller, E Oset and J R Peláez, Phys. Rev. D57, 074001 (1999)

[19] F T Llanes-Estrada, A Dobado, A Gomez Nicola and J R Peláez, Proc. Workshop on Strong and Electroweak Matter 02, (Heidelberg, Germany)

[20] M Gell-Mann and M Levy, Nuovo Cim. 16, 705 (1960)

[21] C S Lim, Implications of sigma models in the standard model and beyond, hep-ph/0010109

[22] V Koch, Int. J. Mod. Phys. E6, 203 (1997)

[23] D Lurié, Particles and fields (Interscience Publishers, London, 1968) p. 266

[24] O Teodorescu, A K Dutt-Mazumder and C Gale, Phys. Rev. C63, 034903 (2001)

[25] G Wolf, B Friman and M Soyeur, Nucl. Phys. A640, 129 (1998)

[26] K Saito, K Tsushima, A Thomas and A Williams, Phys. Lett. B433, 243 (1998)

[27] M Asakawa, C M Ko, P Lévai and X J Qiu, Phys. Rev. C46, R1159 (1992)

[28] G Chanfray and P Schuck, Phys. Rev. C57, 8 (1998)

[29] S H Lee, Phys. Rev. C57, 927 (1998)

[30] T Hatsuda, T Kunihiro and H Shimizu, Phys. Rev. Lett. 82, 2840 (1999)

[31] V Bernard, U-G Meissner and I Zahed, Phys. Rev. Lett. 59, 966 (1987)

[32] P Schuck, Z Aouissat, G Chanfray and J Wambach, nucl-th/0002031

[33] N Grion et al, Nucl. Phys. A763, 80 (2005) 